\title{
ABOUT A PROBLEM OF HERMITE AND BIEHLER
}

\section{TODOR STOYANOV}

(Received 17 July 2000; revised 2 February 2001)

Communicated by P. C. Fenton

\begin{abstract}
A point of departure for this paper is the famous theorem of Hermite and Biehler: If $f(z)$ is a polynomial with complex coefficients $a_{k}$ and its zeros $z_{k}$ satisfy $\operatorname{Im} z_{k}>0$, then the polynomials with coefficients $\operatorname{Re} a_{k}$ and $\operatorname{Im} a_{k}$ have only real zeros.

We generalize this theorem for some entire functions. The entire functions in Theorem 2 and Theorem 3 are of first and second genus respectively.
\end{abstract}

2000 Mathematics subject classification: primary $30 \mathrm{D} 20$.

THEOREM 1. Let $f(z)=c z^{n} \prod_{k=1}^{\infty}\left(1-z / z_{k}\right)$ be an entire function, where $c, z_{k} \in$ $\mathbb{C}, \operatorname{Im} z_{k}>0$. Assume that $\lim _{k \rightarrow \infty}\left|z_{k}\right|=\infty$ and the Maclaurin series of $f$ is $f(z)=\sum_{k=0}^{\infty} a_{k} z^{k}$, where $a_{k}=\alpha_{k}+i \beta_{k}, \alpha_{k}, \beta_{k} \in \mathbb{B}$, and $u(z)=\sum_{k=0}^{\infty} \alpha_{k} z^{k}$, $v(z)=\sum_{k=0}^{\infty} \beta_{k} z^{k}$. Then all roots of $u(z)$ and $v(z)$ are real.

PROOF. The proof is analogous to that for algebraic polynomials.

THEOREM 2. Let $f(z)=c z^{n} \prod_{k=1}^{\infty}\left(1-z / z_{k}\right) \exp \left(z / z_{k}\right)$ be an entire function, where $c, z_{k} \in \mathbb{C}, \arg \left(z_{k}\right) \in(0, \varphi)$, where $0<\varphi<\pi / 2$. Assume that $\lim _{k \rightarrow \infty}\left|z_{k}\right|=\infty$ and the Maclaurin series of $f$ is $f(z)=\sum_{k=0}^{\infty} a_{k} z^{k}$, where $a_{k}=\alpha_{k}+i \beta_{k}, \alpha_{k}, \beta_{k} \in \mathbb{R}$, and $u(z)=\sum_{k=0}^{\infty} \alpha_{k} z^{k}, v(z)=\sum_{k=0}^{\infty} \beta_{k} z^{k}$. Then all roots of $u(z)$ and $v(z)$ satisfy $\arg (z) \notin(\varphi+\pi / 2, \pi)$.

PROOF. We have $f(z)=u(z)+i v(z)=c z^{n} \prod_{k=1}^{\infty}\left(1-z / z_{k}\right) \exp \left(z / z_{k}\right)$. Let $z_{0}$ be such that $v\left(z_{0}\right)=0$ or $u\left(z_{0}\right)=0$. Then

$$
u\left(z_{0}\right)+i v\left(z_{0}\right)=u\left(z_{0}\right)-i v\left(z_{0}\right) \quad \text { or } \quad u\left(z_{0}\right)+i v\left(z_{0}\right)=-\left(u\left(z_{0}\right)-i v\left(z_{0}\right)\right),
$$

(C) 2002 Australian Mathematical Society 0263-6115/2002 \$A2.00+0.00 
that is,

$$
c z_{0}^{n} \prod_{k=1}^{\infty}\left(1-z_{0} / z_{k}\right) \exp \left(z_{0} / z_{k}\right)= \pm \bar{c} z_{0}^{n} \prod_{k=1}^{\infty}\left(1-z_{0} / \bar{z}_{k}\right) \exp \left(z_{0} / \bar{z}_{k}\right)
$$

Suppose that $\arg \left(z_{0}\right) \in(\varphi+\pi / 2, \pi)$; then we prove that

$$
\left|\left(1-\frac{z_{0}}{z_{k}}\right) \exp \left(\frac{z_{0}}{z_{k}}\right)\right|>\left|\left(1-\frac{z_{0}}{\bar{z}_{k}}\right) \exp \left(\frac{z_{0}}{\bar{z}_{k}}\right)\right|
$$

that is,

$$
\left|\exp \left(\frac{z_{0}}{z_{k}}-\frac{z_{0}}{\bar{z}_{k}}\right)\right|>\left|\frac{z_{0}-\bar{z}_{k}}{z_{0}-z_{k}}\right| .
$$

If we let $z_{0}=a+i b, z_{k}=x_{k}+i y_{k}$ and $\bar{z}_{k}=x_{k}-i y_{k}$, where $a, b, x_{k}, y_{k} \in \mathbb{R}, b>0$, $y_{k}>0$, then we have

$$
\begin{aligned}
\left|\exp \left(\frac{z_{0}}{z_{k}}-\frac{z_{0}}{\bar{z}_{k}}\right)\right| & =\exp \left[\operatorname{Re}\left(\frac{z_{0}}{z_{k}}-\frac{z_{0}}{\bar{z}_{k}}\right)\right]=\exp \left[\frac{2 b y_{k}}{x_{k}^{2}+y_{k}^{2}}\right] \quad \text { and } \\
\left|\frac{z_{0}-\bar{z}_{k}}{z_{0}-z_{k}}\right| & =\sqrt{1+\frac{4 b y_{k}}{\left(a-x_{k}\right)^{2}+\left(b-y_{k}\right)^{2}}}
\end{aligned}
$$

Obviously, we have that

$$
\left|\exp \left(\frac{z_{0}}{z_{k}}-\frac{z_{0}}{\bar{z}_{k}}\right)\right|^{2}=\exp \left[\frac{4 b y_{k}}{x_{k}^{2}+y_{k}^{2}}\right]>1+\frac{4 b y_{k}}{x_{k}^{2}+y_{k}^{2}} .
$$

We fix $x_{k}$ and $y_{k}$. Since $\arg \left(z_{0}\right) \in(\varphi+\pi / 2, \pi)$ and $\arg \left(z_{k}\right) \in(0, \varphi)$ we have

$$
\left(a-x_{k}\right)^{2}+\left(b-y_{k}\right)^{2}>x_{k}^{2}+y_{k}^{2} .
$$

Thus we have

$$
1+\frac{4 b y_{k}}{x_{k}^{2}+y_{k}^{2}}>1+\frac{4 b y_{k}}{\left(a-x_{k}\right)^{2}+\left(b-y_{k}\right)^{2}}
$$

which means that $\left|\exp \left(z_{0} / z_{k}-z_{0} / \bar{z}_{k}\right)\right|^{2}>\left|\left(z_{0}-\bar{z}_{k}\right) /\left(z_{0}-z_{k}\right)\right|^{2}$ and the assertion is proved. Hence

$$
\left|c z_{0}^{n} \prod_{k=1}^{\infty}\left(1-\frac{z_{0}}{z_{k}}\right) \exp \left(\frac{z_{0}}{z_{k}}\right)\right|>\left|\bar{c} z_{0}^{n} \prod_{k=1}^{\infty}\left(1-\frac{z_{0}}{\bar{z}_{k}}\right) \exp \left(\frac{z_{0}}{\bar{z}_{k}}\right)\right|,
$$

which is impossible in view of (1). 
THEOREM 3. Let $f(z)=c z^{n} \prod_{k=1}^{\infty}\left(1-z / z_{k}\right) \exp \left[z / z_{k}+\left(z / z_{k}\right)^{2} / 2\right]$ be an entire function, where $c, z_{k} \in \mathbb{C}, \arg \left(z_{k}\right) \in(\varphi+\pi / 3, \pi / 2), 0<\varphi<\pi / 6$. Let $\lim _{k \rightarrow \infty}\left|z_{k}\right|=$ $\infty$ and let the Maclaurin series of $f$ be $f(z)=\sum_{k=0}^{\infty} a_{k} z^{k}$, where $a_{k}=\alpha_{k}+i \beta_{k}$, $\alpha_{k}, \beta_{k} \in \mathbb{R}$ and $u(z)=\sum_{k=0}^{\infty} \alpha_{k} z^{k}, v(z)=\sum_{k=0}^{\infty} \beta_{k} z^{k}$. Then all roots of $u(z)$ and $v(z)$ satisfy $\arg (z) \notin(0, \varphi)$.

PROOF. We have

$$
f(z)=u(z)+i v(z)=c z^{n} \prod_{k=1}^{\infty}\left(1-\frac{z}{z_{k}}\right) \exp \left[\frac{z}{z_{k}}+\frac{1}{2}\left(\frac{z}{z_{k}}\right)^{2}\right] .
$$

Let $z_{0}$ be such that $v\left(z_{0}\right)=0$ or $u\left(z_{0}\right)=0$. Then $u\left(z_{0}\right)+i v\left(z_{0}\right)=u\left(z_{0}\right)-i v\left(z_{0}\right)$ or $u\left(z_{0}\right)+i v\left(z_{0}\right)=-\left(u\left(z_{0}\right)-i v\left(z_{0}\right)\right)$, that is,

$$
\begin{aligned}
& c z_{0}^{n} \prod_{k=1}^{\infty}\left(1-\frac{z_{0}}{z_{k}}\right) \exp \left[\frac{z_{0}}{z_{k}}+\frac{1}{2}\left(\frac{z_{0}}{z_{k}}\right)^{2}\right] \\
& \quad= \pm \bar{c} z_{0}^{n} \prod_{k=1}^{\infty}\left(1-\frac{z_{0}}{\bar{z}_{k}}\right) \exp \left[\frac{z_{0}}{\bar{z}_{k}}+\frac{1}{2}\left(\frac{z_{0}}{\bar{z}_{k}}\right)^{2}\right] .
\end{aligned}
$$

Arguing by contradiction, let us suppose that $\arg \left(z_{0}\right) \in(0, \varphi)$. Then we show that

$$
\left|\left(1-\frac{z_{0}}{z_{k}}\right) \exp \left[\frac{z_{0}}{z_{k}}+\frac{1}{2}\left(\frac{z_{0}}{z_{k}}\right)^{2}\right]\right|>\left|\left(1-\frac{z_{0}}{\bar{z}_{k}}\right) \exp \left[\frac{z_{0}}{\bar{z}_{k}}+\frac{1}{2}\left(\frac{z_{0}}{\bar{z}_{k}}\right)^{2}\right]\right|,
$$

that is,

$$
\left|\exp \left[\frac{z_{0}}{z_{k}}-\frac{z_{0}}{\bar{z}_{k}}+\frac{1}{2}\left(\frac{z_{0}}{z_{k}}\right)^{2}-\frac{1}{2}\left(\frac{z_{0}}{\bar{z}_{k}}\right)^{2}\right]\right|>\left|\frac{z_{0}-\bar{z}_{k}}{z_{0}-z_{k}}\right| .
$$

If we put $z_{0}=a+i b, z_{k}=x_{k}+i y_{k}$ and $\bar{z}_{k}=x_{k}-i y_{k}$, where $a, b, x_{k}, y_{k} \in \mathbb{R}, a>0$, $b>0, x_{k}>0, y_{k}>0$, then we obtain that

$$
\begin{aligned}
& \mid \exp {\left[\frac{z_{0}}{z_{k}}-\frac{z_{0}}{\bar{z}_{k}}+\frac{1}{2}\left(\frac{z_{0}}{z_{k}}\right)^{2}-\frac{1}{2}\left(\frac{z_{0}}{\bar{z}_{k}}\right)^{2}\right] \mid } \\
& \quad=\exp \left\{\operatorname{Re}\left[\frac{z_{0}}{z_{k}}-\frac{z_{0}}{\bar{z}_{k}}+\frac{1}{2}\left(\frac{z_{0}}{z_{k}}\right)^{2}-\frac{1}{2}\left(\frac{z_{0}}{\bar{z}_{k}}\right)^{2}\right]\right\}=\exp \left[\frac{2 b y_{k}}{x_{k}^{2}+y_{k}^{2}}+\frac{4 a b x_{k} y_{k}}{\left(x_{k}^{2}+y_{k}^{2}\right)^{2}}\right]
\end{aligned}
$$

and

$$
\left|\frac{z_{0}-\bar{z}_{k}}{z_{0}-z_{k}}\right|=\sqrt{1+\frac{4 b y_{k}}{\left(a-x_{k}\right)^{2}+\left(b-y_{k}\right)^{2}}} .
$$


Obviously,

$$
\begin{aligned}
\mid \exp & {\left.\left[\frac{z_{0}}{z_{k}}-\frac{z_{0}}{\bar{z}_{k}}+\frac{1}{2}\left(\frac{z_{0}}{z_{k}}\right)^{2}-\frac{1}{2}\left(\frac{z_{0}}{\bar{z}_{k}}\right)^{2}\right]\right|^{2} } \\
& =\exp \left[\frac{4 b y_{k}}{x_{k}^{2}+y_{k}^{2}}+\frac{8 a b x_{k} y_{k}}{\left(x_{k}^{2}+y_{k}^{2}\right)^{2}}\right]>1+\frac{4 b y_{k}}{x_{k}^{2}+y_{k}^{2}}+\frac{8 b^{2} y_{k}^{2}}{\left(x_{k}^{2}+y_{k}^{2}\right)^{2}}+\frac{8 a b x_{k} y_{k}}{\left(x_{k}^{2}+y_{k}^{2}\right)^{2}} .
\end{aligned}
$$

We wish to prove that

$$
1+\frac{4 b y_{k}}{x_{k}^{2}+y_{k}^{2}}+\frac{8 b^{2} y_{k}^{2}}{\left(x_{k}^{2}+y_{k}^{2}\right)^{2}}+\frac{8 a b x_{k} y_{k}}{\left(x_{k}^{2}+y_{k}^{2}\right)^{2}}>1+\frac{4 b y_{k}}{\left(a-x_{k}\right)^{2}+\left(b-y_{k}\right)^{2}},
$$

which will be true if

$$
\frac{1}{x_{k}^{2}+y_{k}^{2}}+\frac{2 b y_{k}}{\left(x_{k}^{2}+y_{k}^{2}\right)^{2}}+\frac{2 a x_{k}}{\left(x_{k}^{2}+y_{k}^{2}\right)^{2}}>\frac{1}{\left(a-x_{k}\right)^{2}+\left(b-y_{k}\right)^{2}},
$$

that is, $\left[\left(a-x_{k}\right)^{2}+\left(b-y_{k}\right)^{2}\right]\left(x_{k}^{2}+y_{k}^{2}+2 b y_{k}+2 a x_{k}\right)>\left(x_{k}^{2}+y_{k}^{2}\right)^{2}$ or

$$
\left(a^{2}+b^{2}\right)\left(x_{k}^{2}+y_{k}^{2}+2 b y_{k}+2 a x_{k}\right)>\left(2 b y_{k}+2 a x_{k}\right)^{2} .
$$

Hence,

$$
\left(3 a^{2}-b^{2}\right) x_{k}^{2}+\left(3 b^{2}-a^{2}\right) y_{k}^{2}+8 a b x_{k} y_{k}-\left(2 b y_{k}+2 a x_{k}\right)\left(a^{2}+b^{2}\right)<0 .
$$

The equation $\left(3 a^{2}-b^{2}\right) x^{2}+\left(3 b^{2}-a^{2}\right) y^{2}+8 a b x y-(2 b y+2 a x)\left(a^{2}+b^{2}\right)=0$ is an equation of a hyperbola. Indeed, if we make the change of the variables

$$
x=\frac{a x^{\prime}-b y^{\prime}}{\sqrt{a^{2}+b^{2}}}, \quad y=\frac{b x^{\prime}+a y^{\prime}}{\sqrt{a^{2}+b^{2}}},
$$

then we have $3 x^{\prime 2}-y^{2}-2 x^{\prime} / \sqrt{a^{2}+b^{2}}=0$. If the angle of rotation is $\psi$, then $\cos \psi=a / \sqrt{a^{2}+b^{2}}$, that is, $\psi \in(0, \varphi)$. Hence

$$
3\left(x^{\prime}-\frac{1}{3 \sqrt{a^{2}+b^{2}}}\right)^{2}-y^{\prime 2}=\frac{1}{3\left(a^{2}+b^{2}\right)},
$$

that is,

$$
\frac{\left(x^{\prime}-1 /\left(3 \sqrt{a^{2}+b^{2}}\right)\right)^{2}}{1 /\left(3 \sqrt{a^{2}+b^{2}}\right)^{2}}-\frac{y^{2}}{1 /\left(\sqrt{3\left(a^{2}+b^{2}\right)}\right)^{2}}=1 \text {. }
$$

After the change $X=x^{\prime}-1 / 3 \sqrt{a^{2}+b^{2}}, Y=y^{\prime}$, we obtain

$$
\frac{X^{2}}{p^{2}}-\frac{Y^{2}}{q^{2}}=1, \quad \text { where } \quad p=\frac{1}{3 \sqrt{a^{2}+b^{2}}}, q=\frac{1}{\sqrt{3\left(a^{2}+b^{2}\right)}} .
$$


Thus $q / p=\sqrt{3}=\tan (\pi / 3)$ and all $w=x+i y$, with $\arg w \in(\varphi+\pi / 3, \pi / 2)$ satisfy

$$
\left(3 a^{2}-b^{2}\right) x^{2}+\left(3 b^{2}-a^{2}\right) y^{2}+8 a b x y-(2 b y+2 a x)\left(a^{2}+b^{2}\right)<0 .
$$

For example, $z_{k}$ satisfy this condition, which confirms the assertion $(*)$. Then we obtain

$$
\left|c z_{0}^{n} \prod_{k=1}^{\infty}\left(1-\frac{z_{0}}{z_{k}}\right) \exp \left[\frac{z_{0}}{z_{k}}+\frac{1}{2}\left(\frac{z_{0}}{z_{k}}\right)^{2}\right]\right|>\left|\bar{c} z_{0}^{n} \prod_{k=1}^{\infty}\left(1-\frac{z_{0}}{\bar{z}_{k}}\right) \exp \left[\frac{z_{0}}{\bar{z}_{k}}+\frac{1}{2}\left(\frac{z_{0}}{\bar{z}_{k}}\right)^{2}\right]\right| .
$$

which contradicts (2). The theorem is proved.

\section{References}

[1] G. Pólya and G. Szegö, Problems and theorems in analysis (Springer, Berlin, 1972).

[2] E. C. Titchmarsh, The theory of functions (Oxford Univ. Press, London, 1939).

Economic University

Department of Mathematics

bul. Knyaz Boris I 77

Varna 9002

Bulgaria

e-mail: todstoyanov@yahoo.com, library@mail.ue-varna.bg 
